\title{
Real-Time and Remote Soil Properties Monitoring with Multi-Sensors
}

\author{
${ }^{1}$ Mohd Nizam Husen, ${ }^{1}$ Mohamad Shahrul Arief Samsudin and ${ }^{2}$ Mohd Kamal Mohd Shah \\ ${ }^{1}$ Universiti Kuala Lumpur, Malaysian Institute of Information Technology, \\ 1016 Jalan Sultan Ismail, 50250 Kuala Lumpur, Malaysia \\ ${ }^{2}$ Advanced Composite and Material Research Group, Faculty of Engineering, \\ Universiti Malaysia Sabah, Jalan UMS, 88450 Kota Kinabalu, Sabah, Malaysia \\ mnizam@unikl.edu.my,+603-2175-4231
}

\begin{abstract}
Soil properties, its features and types are important information for plantation, farming, gardening and any agricultural sectors. Different plants and species requires different soil properties to grow well. Even the same plant might require different soil properties at its different age. The conventional methods of inspecting the soil properties are either taking the soil sample to the lab or bring the measurement device to the site to take the reading. Both methods are time consuming and rather expensive process. This study presents a real-time and remote monitoring of soil features which is essential and very useful for immediate and easy access to the soil information. In this study, readings of the soil condition from sensors could be viewed pervasively, anytime and anywhere, through a mobile application using any handheld device. Among the variables that are measured by the system are soil moisture, temperature and humidity. The system provides real-time data reading and will save it to the database for historical data. The system analyse the data and suggest the suitable plantation for the respective soil properties. The users will also be provided with tips and suggestions to suit their plant's needs.
\end{abstract}

Key words: Soil quality monitoring, soil properties detection, real-time data monitoring, mobile application, suitable plantation, time consuming

\section{INTRODUCTION}

A practice of growing and nurturing plants as part of horticulture is considered as gardening. Ornamental plants are often grown for their flowers, foliage or overall appearance. Useful plants such as root vegetables, leafy vegetables, fruits and herbs are grown for consumption, for use as dyes or for medicinal or cosmetic use. Gardening is considered by many people to be a relaxing activity. Gardening can be a bit complex rather than just watering the plant and checking for bugs or pest control.

With lack of time for gardeners to monitor and manage their garden soils, plants may not grow very well. They could be frustrated due to the reason that they cannot manage their garden very well. With the proposed mobile application, it will help new gardeners or even master gardeners to monitor their plants effectively by monitoring the soil suitability to grow certain type of plants. In Malaysia, the same current value of gardening growth in 2016 as in 2015 (GVR., 2019). Many factors such as the slowing of economic conditions in Malaysia affect gardening, since, the costs of this activity are relatively low. People were also keen to participate in gardening as a leisure activity as well as to decorate their homes with some flora. Besides that, the global gardening equipment market size was valued at USD 74.1 billion in 2015 and expected to increase more in the future (Anonymous, 2016).

Factors affecting plant growth: Gardening as it seems might be simple but contain many factors that may make the plant healthy. There are four concerning factors that affect plant growth which is light, water and humidity, temperature and nutrients. These four factors may affect the plant's growth which is making the plant grow more quickly or more slowly. Lack any of the four can cause the plant stress which stunts or changes growth. It is important to learn about each of the four factors and what the plant needs to maximize plant growth factors for a healthy garden.

Corresponding Author: Mohd Nizam Husen,Universiti Kuala Lumpur, Malaysian Institute of Information Technology, 1016 Jalan Sultan Ismail, 50250 Kuala Lumpur, Malaysia, mnizam@unikl.edu.my, +603-2175-4231 
As an example, a chrysanthemum plant is a short-day plant which it requires long days or vegetative growth and short days for flowering. During the vegetative growth, it requires long day with bright sunlight and high temperature. But during its flowering stage, it requires short day and low temperature. The relative humidity of $75-90 \%$ is suitable for this plant. The optimal soil $\mathrm{pH}$ range for the growth is 6.5-7.5 (Anonymous, 2018a).

Another example is for a hibiscus plant which needs warm and steady temperature to grow ranging from 18-28 Celsius. Hibiscus plant needs at least $6 \mathrm{~h}$ of sunlight per day to grow. It also need moist and welldrained soil for growth. Potassium promotes the plant's growth and metabolism which by promoting photosynthesis for the plant. Meanwhile, the hibiscus needs suitable amount of phosphorous and nitrogen. Over exceeding the dosage can damage the plant (Anonymous, 2018b).

Sensors for soil properties monitoring: There is a certain variable that can be monitored using sensors that may contribute to a healthy plant's growth. For example, we can use the moisture sensor to detect the moisture of the soil. Soil moisture sensors measure the volumetric water content in the soil which is then converted to readings, so that, we can see the moisture of the soil.

Another sensor that we can use for gardening is the temperature sensors. Surrounding temperature may give significant effect on the plant. By using a temperature sensor, we can see if the temperature is suitable for the plant or not. Certain plant may need lower temperature to survive such as broccoli, cabbage or carrots (Anonymous, 2018).

Meanwhile, Ehsan et al. (2019) build a portable environmental monitoring system which collect data from environment using sensors to detect soil pollution. Another notable sensor that can be used to measure the soil quality is $\mathrm{pH}$ and electrical conductivity sensors. $\mathrm{pH}$ sensor measures the acidity of the soil, so that, the soil is suitable for certain type of plants, electrical conductivity sensors do it job by measuring the salinity of the soil which is a parameter that indicates whether the soil needs fertilizer or not. Skierucha et al. (2012) monitor soil moisture with simultaneous measurement of soil temperature and electrical conductivity.

As for this project, two of this parameters were used which are temperature and moisture. This is because these are the most important features to know the soil properties for gardening and farming. Furthermore, we are using Wi-Fi as the medium of communications as opposed to (Peijin et al., 2011) which used Zigbee.

\section{MATERIALS AND METHODS}

To design and develop the proposed real-time soil properties monitoring system, a number of important soil properties parameters were selected based on (GVR., 2019; Anonymous, 2016; Gunawan et al., 2019). The parameters that have been experimented is moisture and temperature.

Parameters: There are two parameters under study in this research.

Moisture: The soil of the garden needs to be an intermediate condition for the plant to grow, not too dry and not too wet. Gardeners may not know this thing, especially, the new one. So, in the project, soil moisture is one of the important parameters.

Temperature: Another thing for gardeners to monitor is temperature. For instance, a different type of plant may need a different type of temperature. One of the obvious effects of heat during photosynthesis. For example, tomatoes get into trouble when the temperature exceeds $36^{\circ}$ celsius.

Design and development: In this study, we design and develop two main components of the project:

Hardware design: The hardware that have been used in the development of the system are a moisture sensor, a temperature sensor which can detect air humidity and surrounding temperature, Arduino Uno board, a $16 \times 2$ LCD display and ESP-01 which is the Wi-Fi transceiver.

Mobile application design: The mobile application also must be developed as a medium for the user to view the parameters live. The software that have been used in the development of the system are Android Studio, Arduino IDE and ThingSpeak. Arduino IDE is a compiler that produces binary machine code for the target processor. It provides a development environment for their microcontrollers. Android Studio is a platform for writing down the codes required for the mobile application graphical user interface and functionality. Thingspeak is an IOT-platform to receive the sensor readings and display it in a graphical view.

Setup and testing: The setup of this development was being depicted as in Fig. 1 and 2. The hardware was tested by using a container of soil which was differentiated by 


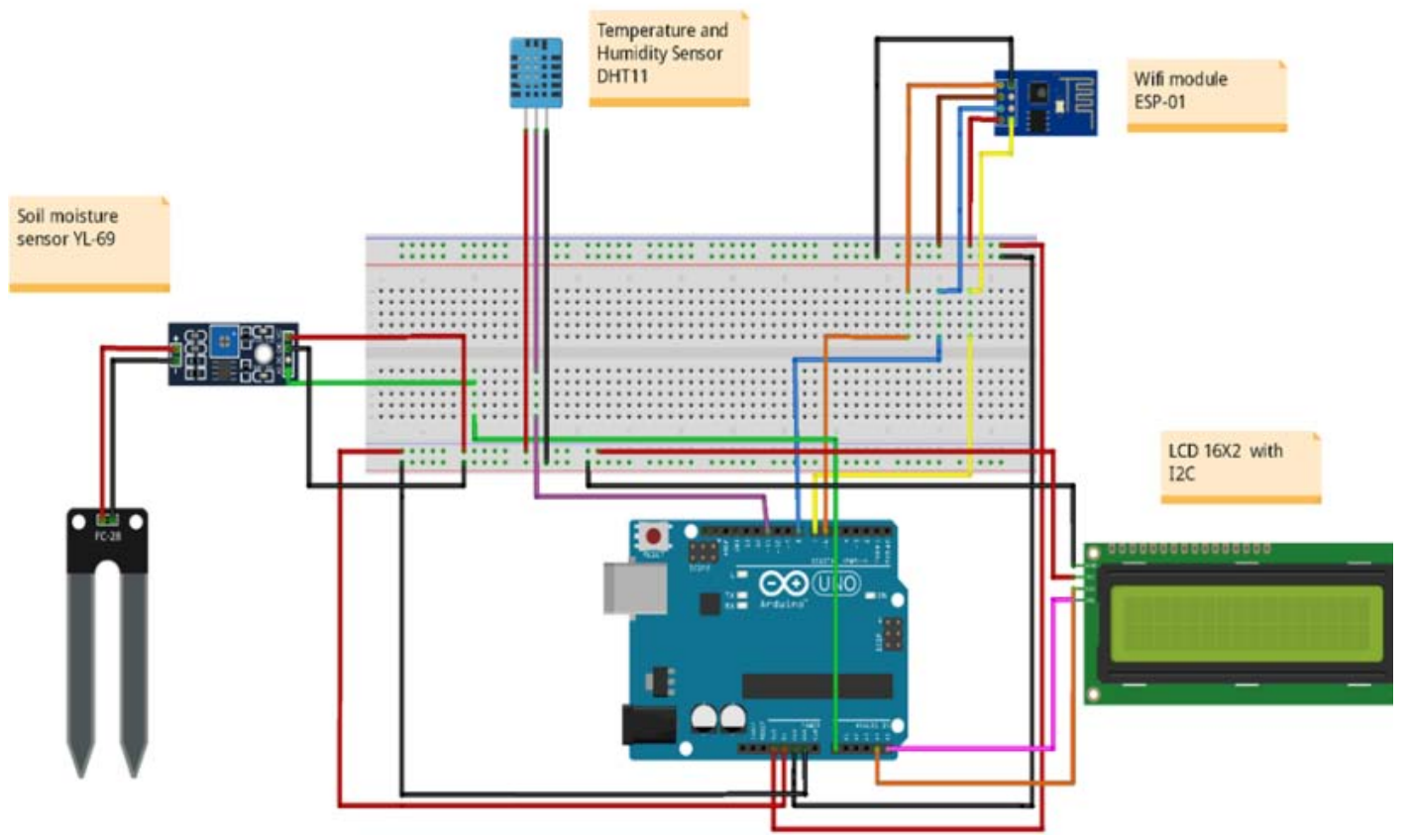

Fig. 1: Hardware interface design diagram

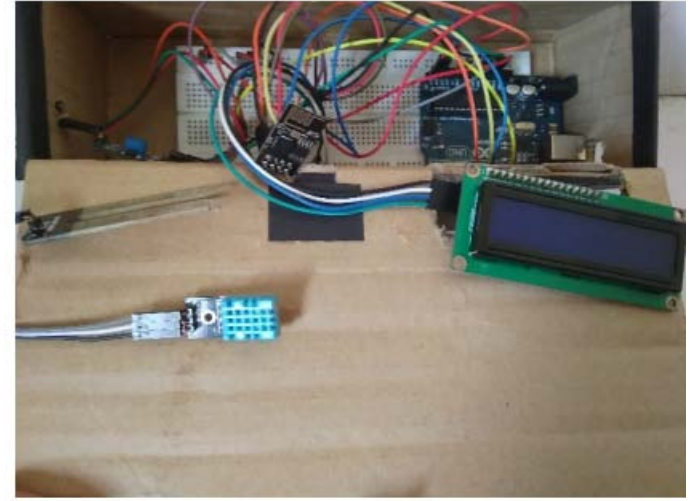

Fig. 2: Hardware interface design

the moisture content. The room also must be in a different temperature as one of the parameters involved was room's temperature.

If any one of the parameters fall outside the safety quality range, the soil quality is considered unsafe and the LCD display will display a message to the user for further action. The examples of the testing output are shown in Fig. 3.

Figure 4 shows the remote monitoring by the user; the output on the LCD is also available in a mobile-based system that displays the information of the soil quality parameters in real-time. It also provides suggestion based on current temperature.

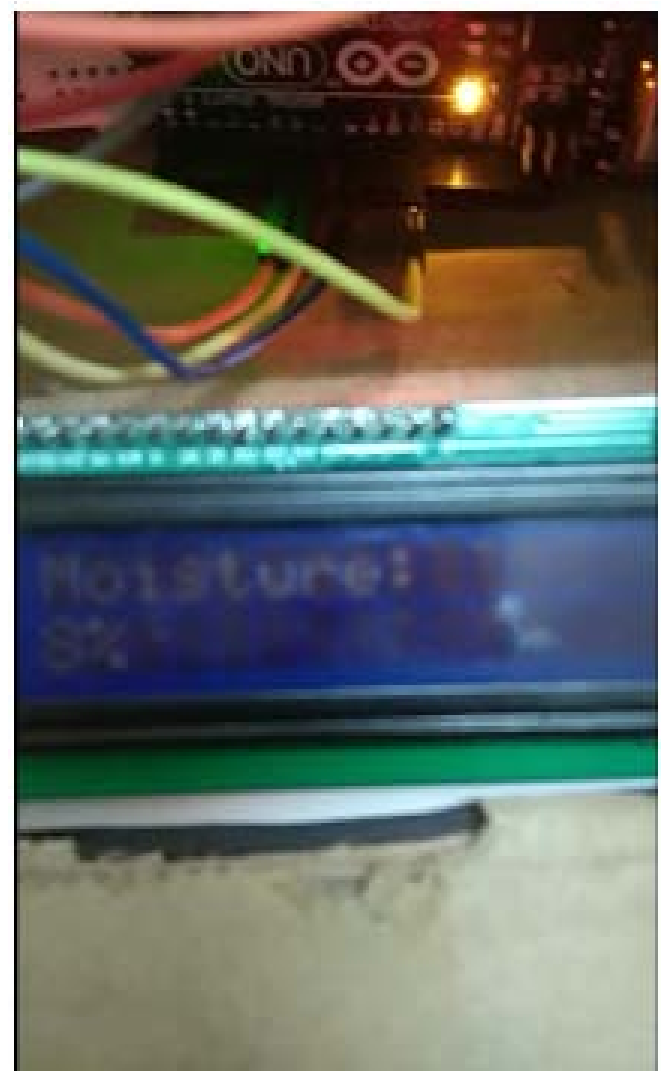

Fig. 3: Example of low moisture indication 


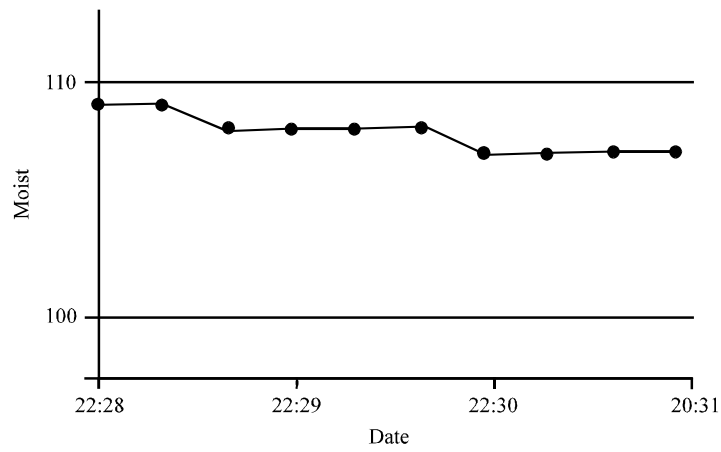

Fig. 4: Mobile application interface. Current moisture: 107.0, suggestion: so much water leave the plant for a few days before watering it again

\section{RESULTS AND DISCUSSION}

This study elaborates the performance test results from our experimental study which includes the soil properties detection performance based on each soil parameter and the performance of the wireless communication between the sensing node and the mobile application.

Performance by parameters: A total of 572 testing of different soil conditions for known true values have been implemented during the testing. Figure 5 shows the Receiver Operating Characteristics (ROC) curves which representing the overall results on the performance of the system by parameters. As indicated in the figure, an average of $98 \%$ is achieved for both parameters.

Detections both by moisture and temperature show very good performance with 99 and $97 \%$, respectively. The suggestion of action provided by the system also perform very well with $100 \%$ accuracy. The detection results match the actual soil properties which were premeasured.

Performance of wireless communication: The wireless communication between the sensors measurement node and the server, followed by accessing the database from the mobile application using Wi-Fi transmitter and receiver have been tested. As illustrated in Fig. 6, the results obtained were $99.3 \%$ of success rate from 572 tests. This high percentage performance shows that Wi-Fi is a suitable medium to be used as the wireless communication to transmit data that has been collected by the sensors to the server. This result corroborates the result by Husen and Lee (2016).

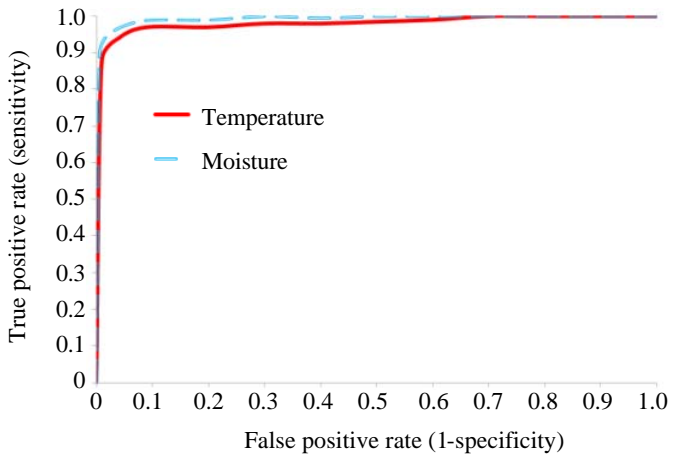

Fig. 5: The ROC curves representing the performance of the proposed soil properties monitoring by parameters

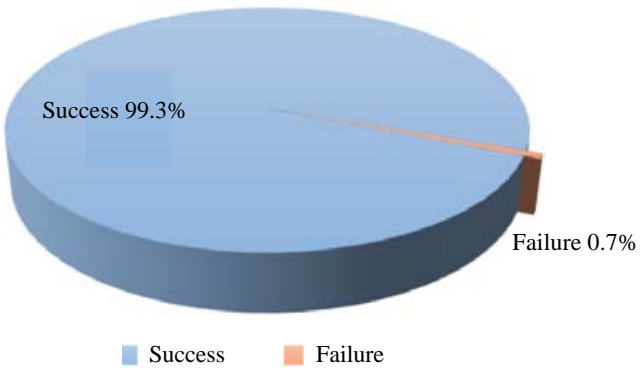

Fig. 6: The result of the wireless communication testing shows that a very good percentage of $99.3 \%$ is achieved through Wi-Fi medium

\section{CONCLUSION}

The moisture and temperature of soil are important parameters in determining the suitability of soils to certain plants. In this study, both parameter values have been measured by respective sensors and being processed. The readings are then sent to the server through wireless communication. The readings are able to be viewed in real-time and remotely from the developed mobile application. This application is significance to gardeners, farmers, landscapers or researchers because of the ubiquitous and real-time access capability. Future work could include the design and implementation of electrical conductivity and $\mathrm{pH}$ sensors as those are also important parameters.

\section{ACKNOWLEDGEMENT}

The research work presented in this research was partly supported by the Universiti Kuala Lumpur-Short Term Research Grant (STR17021). 


\section{REFERENCES}

Anonymous, 2016. Gardening equipment market analysis. Grand View Research, Pune, India.

Anonymous, 2018a. Chrysanthemum cultivation information guide. AgriFarming, Hyderabad, India. https://www agrifarming.in/chrys anthemumcultivation/

Anonymous, 2018b. Flower Farming. AsiaFarming, USA. https:/www asiafarming.com/hibis cus-farmingindoors-information/

Ehsan, M.S., S. Biswas, A. Nawar and R. Siddiqua, 2019. Portable environmental monitoring system. BSc Thesis, BRAC University, Bangladesh.

GVR., 2019. Gardening equipment market analysis by product (hand tools, lawnmowers, trimmers \& edgers, water management equipment), by end-use (residential, commercial), by region (North America, Europe, APAC, LATAM, MEA) and segment forecasts, 2018-2025 (2016). Grand View Research, Pune, India. https://www.grandviewresearch. com/industry-analysis/gardening-equipment-market
Gunawan, R., T. Andhika, Sandi and F. Hibatulloh, 2019. Monitoring system for soil moisture, temperature, $\mathrm{ph}$ and automatic watering of tomato plants based on internet of things. Telekontran, 7: 66-78.

Husen, M.N. and S. Lee, 2016. High performance indoor location Wi-Fi fingerprinting using invariant received signal strength. Proceedings of the 10th International Conference on Ubiquitous Information Management and Communication (IMCOM'16), January 4-6, 2016, ACM, Danang, Viet Nam, ISBN:978-1-4503-4142-4, pp: 42-1-42-6.

Peijin, H., J. Ting and Z. Yandong, 2011. Monitoring system of soil water content based on Zigbee wireless sensor network. Trans. Chin. Soc. Agric. Eng., Vol. 2011, 10.3969/j.issn.10026819.2011 .4 .040

Skierucha, W., A. Wilczek, A. Szyp³ owska, C. S3awinski and K. Lamorski, 2012. A TDR-based soil moisture monitoring system with simultaneous measurement of soil temperature and electrical conductivity. Sens., 12: $13545-13566$. 